\title{
Is Dark Matter Made up of Xons?
}

\author{
Jean-Paul Auffray \\ ex Courant Institute of Mathematical Sciences, New York University, New York, USA \\ Email: jpauffray@yahoo.fr
}

Received June 5, 2013; revised July 8, 2013; accepted August 1, 2013

Copyright (C) 2013 Jean-Paul Auffray. This is an open access article distributed under the Creative Commons Attribution License, which permits unrestricted use, distribution, and reproduction in any medium, provided the original work is properly cited.

\begin{abstract}
Three months before his untimely death in Paris in July 1912, Henri Poincaré formulated the conjecture that Planck's action element could (should) be regarded as constituting a "véritable atome", i.e. an "atom of motion", whose integrity arises from the fact that the "points" it contains are equivalent to one another from the standpoint of probability. In this paper we investigate the possibility that this conjecture provides a clue to the origin and nature of dark matter.
\end{abstract}

Keywords: Smolin; Poincaré; Action; Space; Vacuum; Scale Relativity; Nottale; Dark Matter; Xons

\section{Introduction}

Some years back, in "The Trouble with Physics", Lee Smolin wrote: "Many quantum-gravity theorists believe there is a deeper level of reality, where space does not exist (this is taking background independence to its logical extreme)". In Lee Smolin's conception "background independence" refers to the proposal that "the laws of nature can be specified completely without making any prior assumption about the geometry of space" and to the requirement that "space and time emerge from the laws rather than providing an arena in which things happen". To achieve this objective, he offered this advice (his italics): "Don't start with space, or anything moving in space." He proposed, instead: "Start with something that is purely quantum-mechanical and has, instead of space, some kind of purely quantum structure [1]."

It is noteworthy that, in this statement, Lee Smolin refers to space - and not to time (nor to spacetime) - as the ingredient one should dispose of, suggesting to replace it by something having a "purely quantum structure". There is a reason for this distinction: quantum gravity specialists have argued successfully that "the best strategy for understanding quantum gravity is to build a picture of the physical world where the notion of time plays no role at all". In his recently published review article "Forget time", Carlo Rovelli has shown convincingly how this can be done [2].

We attempt in this Note to provide a plausible answer to the request Lee Smolin has formulated concerning space. Our scheme is founded in a (little known) action conjecture originally formulated by Henri Poincaré that we shall now describe.

\section{Premises}

\subsection{Poincaré's (Little Known) Action Conjecture}

Leibniz's invention of his Dynamica in 1689, at the heart of which he placed the abstract concept of action (actio), provided an alternate framework to Newton's System of the World in which Absolute Space and Absolute Time enter as primary or "God-given" Principles. The subsequent discovery of the Principle of Least Action, followed by the invention of the elementary quantum of action by Max Planck in 1900, placed action at the very heart of modern physics.

As a physical entity worthy of consideration per se, the elementary quantum of action was investigated by Henri Poincaré shortly before his untimely death in July 1912 when he proposed that it constitutes a "véritable atome"-an "atom of motion"-whose integrity arises from the fact that the "points" it contains are equivalent to one another from the standpoint of probability, a highly significant remark [3]..

\subsection{Origin of the Poincaré Action Conjecture}

Poincaré's action conjecture has its roots in a remark originally made by Max Planck concerning his discovery of a connection between the quantum of action and the Liouville Theorem of classical physics reformulated in the framework of Gibbs' statistical mechanical method of representations in phase space. Poincaré apparently first heard of the connection when he met Planck at the Conseil de Physique ("First Solvay Congress") gathered in Brussels in October-November 1911. Transcripts of the Conseil meetings show that Poincaré was inquisitive of 
the true nature of Planck's action element all through that week [4].

The issue divided the Conseil into three "camps": those, a majority, who did not care one way or the other; those, a minority led by Arnold Sommerfeld, who considered Planck's action element to be the true fundamental entity one needed to take into account in the formulation of emerging quantum theories; and those, led by Albert Einstein, who favored the "energy quantum" over the action element.

Obviously impressed by Planck's connection of the action element with the Liouville Theorem and by Sommerfeld's vigorous defense of the deep physical significance he attached to this connection, Poincaré formed his conjecture shortly after the Conseil ended. To clarify the issue involved, we shall briefly outline how the connection with the Liouville Theorem arises.

\subsection{Connection with the Liouville Theorem}

Consider two allowed states of a given (classical) physical system. If one of the states is a necessary consequence of the other, then the two states are equally probable. Let $q$ represent a generalized coordinate of the system and $p$ the corresponding conjugate momentum, then $d p d q$ constitutes an infinitesimally small elementary domain of probability associated with the system. Planck's quantum hypothesis consists in assuming that, rather than being infinitesimally small, the elementary domains are all equal and given by the relation $\iint \mathrm{d} p \mathrm{~d} q=h$, where $h$ is Planck's famous "second universal constant" (the first is $k$, which has the dimension of entropy and intervenes to make $k \mathrm{~T}$, where $\mathrm{T}$ is the system temperature, an energy).

\subsection{Poincaré Invents the Concept of Quantum Jumps}

Poincaré's reasoning concerning the structure of Planck's finite domains is striking: "These domains are indivisible, he wrote. If we know the system to be in one of them, then everything is automatically determined. If it were not, if events that are to follow were not fully determined by that knowledge - in a word, if they were to differ depending on the system being in such or such part of the domain - then, since the probability of some future events would not be the same in its diverse parts, the domain considered would not be indivisible from the viewpoint of probability." And he concluded: "This means that all the system states that correspond to a given domain are undistinguishable from one another and therefore constitute one and the same state." A conclusion that led him to assert unambiguously: "We are therefore led to formulate the following fundamental theorem: A physical system can exist only in a finite number of possible distinct states; it jumps from one of these states to another without passing through intermediate states [5]."

Thus was born in 1912, under Poincaré's pen, the concept of quantum jumps.

\subsection{Transition}

In what follows we examine the possibility that Planck's action element, understood in the sense proposed by Poincaré, should indeed be regarded to be not simply a "universal constant"-Planck's constant" akin to other "universal constants" such as $\pi$, e, c or $\mathrm{k}$-but as the fundamental principle of quantum physics (we use here the word principle in the Aristotelian sense it originally had: "that which comes first" [archē]).

To implement this scheme, we shall engage in a line of thought initiated by James Gray, Yang-Hui He, Vishnu Jejjala and Brent D. Nelson in their paper "Vacuum Geometry and the Search for New Physics" in which they explore the possibility of finding a "hidden structure" in vacuum space [6].

\section{The Concept of Space Revisited}

Ordinary space is thought (is said) to be composed of dimensionless "points"-0-branes in M-theory-whose (sole) characteristics is to "exist". In Euclidian space, and in relativistic spacetimes as well, these points are related to one another via notions of proximity and distance (interval).

We postulate instead the presence in nature of a vacuum space containing:

1) An unstructured chaotic or "passive" substrate composed of dimensionless elements we shall call "i-points" pending further examination of their characteristics. ipoints present in the substrate bear no geometrical relationships with one another.

2) A primitive or active principle that we shall call the xon and designate as $h$, since, as we shall show, it corresponds to Planck's 1900 invention of the action element.

In what follows, we shall call the interaction of xons with i-points the "occurrence" of $h$ in the chaotic substrate and we shall say that the result of the occurrence is the potential formation in the substrate of i-point clusters, designation which conveys no predetermined algebraic or geometric connotations.

\section{The Crux of the Matter}

If Nature's underlying reality is indeed to be described in terms of a passive substrate which acquires geometric and/or dynamic properties through the occurrence of an action element acting in its midst as a "first principle" or cause, then significant consequences must ensue. One in particular is that the symbol $h$, which represents the ac- 
tion element, should no longer be considered to designate simply a "universal constant of nature" akin to other universal constants such as $\pi$, e or $\mathrm{c}$, but as representing a physical entity possessing characteristics in its own rights.

To implement this requirement, we shall make use of two fundamental axioms concerning action proposed by Leibniz in his legendary 1688 treatise, Dynamica de Potentia:

Axiom 1. "One can understand what action in a body is only in an indirect way";

Axiom 2. "Motion's formal actions are the results of the composition of diffusions and intensions [7]".

Implementing these two axioms, we seek representations of $h$ in terms of the composition of a diffusion or extension, $l$, and an intension or intensity, $p$, yielding the fundamental equation

$$
l p=h .
$$

If we assume the "extension" $l$ thus introduced to represent a "length" of some sort, then this equation recovers Louis de Broglie's 1923 relation $p=h / \lambda$ which associates a wavelength $\lambda$ to an electron moving with momentum $p$. In our scheme, however, the extension thus introduced assumes an entirely different significance: it represents a "geometric structure" of some kind induced in the i-points substrate by the active principle, $h$.

The De Broglie relation, which we wrote as $l p=h$, may also be written in the alternative form

$$
E d=h
$$

where $E$ measures an energy and $d$ designates a "timelike" extension or "duration". This recovers the photon relation $E=h v$.

Any physical theory must ultimately be related to what one might want to call "reality as classical physicists see it" which constitutes a particular representation or interpretation arising from the underlying reality. For the purpose of the present demonstration, we take the view that "reality as classical physicists see it" depends primarily on the assumed validity of the Euclidian geometry axiom according to which there exist in nature straight line segments whose lengths can be measured by means of fixed-lengths rods taken as units - a method, incidentally, Albert Einstein used, and later rejected, in his first 1905 paper on Special Relativity.

\section{From Chaos to Fractal Geometry}

On this basis, we seek to establish a connection between the assumed underlying chaotic "reality" in which action is assumed to be the active ingredient and ordinary spacetime physics.

There is indeed no compelling reason to assume that $h$-induced extensions - $l$ or $d$-relate to "Euclidian-type" structures. We shall assume instead that they relate at best to fractal structures.

To explore this idea we shall borrow extensively from Laurent Nottale's Theory of Scale Relativity [8].

Let $D$ be the fractal dimension and let $\delta$ designate the resolution dimension defined as the underlying fractal dimension minus the topological (observable) dimension: $\delta=D-D_{T}$. Nottale has called the resolution dimension $\delta$ thus defined the system "fifth dimension" or "djinn".

The use of the word resolution in this context refers to the following. The relations $l p=h$ and $E d=h$ allow any value of $l$ or $d$ to occur. When particular values prevail, we will say, following Nottale, that they specify the cluster state of resolution. This assigns to the cluster a new kind of coordinate which is neither space-like nor time-like but reflects instead a fundamental dependence of the cluster geometry on resolution.

To specify more closely the significance of this concept, we shall reason concurrently within two frameworks: 1) the framework that arises from our 2-principle underlying scheme; and 2) the framework of ordinary four-dimensional spacetime physics in which distances between events (world-points) are the relativistic intervals that connect them. To simplify the presentation however, we shall use the word "length" rather than the word "interval" to designate distances between events (points) in spacetime.

\subsection{Measuring Distances in Spacetime}

Distances can be measured in spacetime only if one disposes of "yardsticks" to make the measurements.

Let the extension $l$ associated with an $h$-induced $i$ points cluster be $l(\varepsilon)$ when the yardstick length is $\varepsilon$ in spacetime. In the framework of his Scale Relativity Theory (SR), Laurent Nottale has shown that $l(\varepsilon)$ tends to infinity when $\varepsilon$ tends to zero. The yardstick length $\varepsilon$ is only defined relatively to the length $\varepsilon$ ' of another yardstick, however. In the simplest formulation of the theory, the resolution dependence of $l$ assumes the simple form $l^{\prime}=\left(\varepsilon / \varepsilon^{\prime}\right)^{\delta} \cdot l$. When $\delta=1$, which arises when the fractal dimension is 2 and the topological dimension is 1 , the resolution dependence becomes $l^{\prime} / l=\varepsilon / \varepsilon^{\prime}$. Inasmuch as it corresponds to the supposition $t^{\prime} / t=1$ which characterizes classical mechanics, the supposition $\delta=1$ may be said to impart to this formulation a Galilean character. In a more sophisticated formulation of the theory a transition scale $\varepsilon_{0}$ occurs such that for $\varepsilon \ll \varepsilon_{0}$ the system is fundamentally resolution-dependent, while for $\varepsilon \gg \varepsilon_{0}$, it becomes essentially resolution-independent. The transition scale thus defined establishes a connection between quantum and classical behavior, a highly desirable feature for a scheme that seeks to define a new kind of background for M-Theory brane propagation and interactions. To help visualize what is involved, we shall out- 
line a representation of the process even though it is not strictly applicable to the proposed scheme since it involves geometrical concepts not explicitly present in the scheme. It is based on original work by Polish mathematician Waclaw Sierpinski. In constructing this illustrative example we selected on purpose the group-theoretical concept of "substitution" to designate the self-ordering process: being equivalent to one another, i-points can be substituted to one another without disrupting the cluster integrity

\subsection{A Visual Aid}

Let $\mathrm{ABC}$ designate an equilateral triangle supposedly representing an $h$-induced i-points cluster. "Distances" between any two i-points in this cluster are not defined. Nevertheless, for the purpose of the demonstration, let us assume that for each i-point in the cluster distances are defined with respect to the three arbitrarily selected i-points $\mathrm{A}, \mathrm{B}$ and $\mathrm{C}$ which define the cluster "boundaries". Let 1 designate an i-point chosen randomly within the cluster. Consider the substitution that replaces the i-point 1 by the i-point 2 located at mid-distance between 1 and $B$. Let a new substitution replace the i-point 2 thus identified by the i-point 3 located at mid-distance between i-point 2 and i-point $\mathrm{C}$. Each subsequent substitution advances the original i-point midway in the direction of $\mathrm{A}, \mathrm{B}$ or $\mathrm{C}$, the direction being chosen at random. Somewhat surprisingly, if pursued ad infinitum, this process transforms the random distribution of i-points in the original cluster into a fractal distribution of i-points resembling a Sierpinski triangle. This construction illustrates the fact that a primitive form of self-organization can result from a (partially) random substitution process occurring within an undifferentiated substrate [9].

\section{Esoteric Representations of $\boldsymbol{h}$}

In Quantum Electrodynamics (QED), the electron action is written as

$$
S=2 \pi \cdot(l p-E d+\varphi \sigma+e \chi) .
$$

In this formula, besides $l p$ and $E d$, two additional representations of the action element occur: $\varphi \sigma$ and $e \chi$, where $\varphi$ measures an angle, $\sigma$ the corresponding conjugate angular momentum (an action), and where $e$ represents the electron charge and $\chi$ designates a gauge function.

In Scale Relativity, the mysterious gauge function $\chi$ which occurs in this formula is identified with the logarithm of the resolution scale factor $\rho=e^{\prime} / e$. Recalling that, in our notation, $\varepsilon_{0}$ designates the transition scale such that for $\varepsilon \ll \varepsilon_{0}$ the system considered is fundamentally resolution-dependent, while for $\varepsilon \gg \varepsilon_{0}$, it becomes essentially resolution-independent, let us consider the case when $\ln \rho=\ln \left(\varepsilon_{0} / \varepsilon\right)$. Nottale introduces here in his theory the postulate of the uniformity of scale space in general and of the gauge function

$$
\chi=\ln \rho=\ln \left(\varepsilon_{0} / \varepsilon\right)
$$

in particular. When this is done, the gauge function is found to be conjugate to the electron charge and the electron charge is quantized, an interesting result.

There is more. A fifth representation of $h$ arises from the relation $e^{2} / 2 \alpha c=h$ which connects $h$ with the electron charge $e$, the fine structure constant $\alpha$, a pure number, and the limit velocity $c$ that occurs in Poincaré's relativistic electron dynamics. In this representation, the part that constitutes Leibniz's diffusio factor is not easily identified. In the relation $e^{2} / 2 \alpha c=h, e$ can vary only if the fine structure constant $\alpha$ varies accordingly.

Other representations of the action element will ensue if one writes other known quantum mechanical relations in the form "something $=h$ ". Each new representation provides a particular insight into the complex and deep nature of the action element.

\section{Conclusions}

In our fundamental relation $l p=h$, if $l$ has the dimension of a length, then $p$ has the dimension of a momentum, $p=$ $m v$, thus introducing in the scheme a (hidden) velocity $v$ and a (hidden) mass, $m=p / v$.

At the 25th Solvay Congress held in Brussels in October 2011, Rapporteur Juan Maldacena reviewed the problems attached to current research in Quantum SpaceTime physics. One of them relates of course to the need to identify the nature and origin of the mysterious dark matter and dark energy which seem to pervade the known Universe [10].

Henri Poincare formulated his action conjecture in the wake of the First Solvay Congress held in the same city one hundred and two years ago. If, as he suggested, Max Planck's action element $h$ is indeed a veritable atome-a xon in our proposed terminology - and if the scheme we have constructed in these pages to accommodate this conjecture has any kind of vraisemblance, then it would follow that vacuum space might indeed be the site of complex hidden activities ensuing from the presence in its midst of xons acting on i-points to create clusters and yield, in particular... some form of Dark Matter?

\section{Acknowledgements}

We wish to express our gratitude to Laurent Nottale and to Cédric Villani for their precious comments on certain aspects of this work and, retrospectively, to Louis de Broglie for his encouragements in the early phase of this research and to Professor Richard Feynman who inspired us to coin the word xon [11]. 


\section{REFERENCES}

[1] L. Smolin, "The Trouble with Physics," Houghton Mifflin, Boston, 2006.

[2] C. Rovelli, "Forget Time," Essay Written for the FQXi Contest on the Nature of Time, 2008.

[3] H. Poincaré, "Mathematics and Science: Last Essays," Dover, New York, 1963.

[4] "La Théorie du Rayonnement et les Quanta," Transcripts of the Solvay Congress 1911, Gauthier-Villars, Paris, 1912.

[5] H. Poincaré, "Dernières Pensées," Op. Cit., p. 185.

[6] J. Gray, Y.-H. He, V. Jejjala and B. D. Nelson, "Vacuum Geometry and the Search for New Physics," 2006. arXiv:hep-th/0511062v2
[7] G. W. Leibniz, "Actiones Motuum Formales Sunt in Ratione Composita Diffusionum et Intensionum," Mathematische Schriften 6, Georg Olms Verlag, Hildesheim, New York, 1971, p. 355.

[8] L. Nottale, "Scale Relativity and Fractal Space-Time: A New Approach to Unifying Relativity and Quantum Mechanics," Imperial College Press, London, 2011.

[9] H.-O. Peitgen et al., "Fractals for the Classroom," SpringerVerlag, Berlin, 1993, pp. 49-60.

http://dcr.rpi.edu/commdesign/class $1 . h t m l$

[10] J. Maldacena, "The Quantum Space-Time," www.sns.ias.edu/ malda/Solvay2011.ppt

[11] L. Lederman and D. Teresi, "The God Particle," Delta Books, New York, 1993, p. 211. 